\title{
New Explicit Exact Solutions of the One-Dimensional Parabolic-Parabolic Keller-Segel Model
}

\author{
Lihua Zhang*, Lixin Ma, Fengsheng Xu \\ Department of Mathematics, Dezhou University, Dezhou, China \\ Email address: \\ zzlh100@163.com (Lihua Zhang) \\ ${ }^{*}$ Corresponding author \\ To cite this article: \\ Lihua Zhang, Lixin Ma, Fengsheng Xu. New Explicit Exact Solutions of the One-Dimensional Parabolic-Parabolic Keller-Segel Model. \\ Applied and Computational Mathematics. Vol. 7, No. 2, 2018, pp. 50-57. doi: 10.11648/j.acm.20180702.13
}

Received: January 30, 2018; Accepted: February 11, 2018; Published: March 7, 2018

\begin{abstract}
One-dimensional parabolic-parabolic Keller-Segel (PP-KS) model of chemotaxis is considered. By using the generalized tanh function method, $\left(G^{\prime} / \mathrm{G}\right)$-expansion method and variable-separating method, plenty of new explicit exact solutions, including travelling wave solutions and non-travelling wave solutions, are obtained for the PP-KS model. Compared to the existing results, more new exact solutions are derived and the obtained solutions all have explicit expressions.
\end{abstract}

Keywords: Keller-Segel Model, Generalized Tanh Function Method, ( $\left.G^{\prime} / G\right)$-Expansion Method, Variable-Separating Method, Exact Solutions

\section{Introduction}

Investigating exact solutions of nonlinear evolution equations plays an important role in nonlinear science. For example, the wave phenomena observed in fluid dynamics, plasma and elastic media are often modelled by bell-shaped sech solutions and kink-shaped tanh solutions. The effort to find these solutions is significant for the understanding of many phenomena in physics, chemistry and biology, because they may give more quantitative information. In the past several decades, many effective methods for obtaining exact solutions of nonlinear partial differential equations (NLPDEs) have been presented such as Hirota's bilinear method [1], inverse scattering method [2], Backlund transformation method [3], Painleve expansion method [4], Jacobi elliptic function expansion method, the new generalized algebraic method and so on [5-10].

The prototypical chemotaxis model was proposed by Keller and Segel in the 1970s to describe the aggregation of cellular slime molds Dictyostelium discoideum in response to the chemical cyclic adenosine monophosphate [11-12]. In its general form, Keller-Segel model reads

$$
\left\{\begin{array}{c}
u_{t}=\nabla \cdot(d \nabla u-\chi u \nabla \phi(v)), \\
v_{t}=\alpha \Delta v+f(u, v),
\end{array}\right.
$$

where $u$ and $v$ denote the cell density and chemical concentration, respectively. $d>0$ and $\alpha \geq 0$ are cell and chemical diffusion coefficients, respectively. $\chi>0$ is called the chemotactic coefficient measuring the strength of the chemical signal. Here $\phi(v)$ is referred to as the chemosensitivity function describing the signal detection mechanism and $f(u, v)$ is a function characterizing the chemical growth and degradation. When $\phi(v)=\ln v, f(u, v)=-k u$,

Keller and Segel [13] performed theoretical analysis of the one-dimensional form of (1) to interpret the propagating travelling bands of bacterial chemotaxis experimentally observed in $[14,15]$. Since then, the study of travelling wave solutions to (1) has received extensive attentions [16-17] and the references quoted therein. The readers are referred to [18] and $[19,20]$ for more detail about biological motivation and mathematical introduction of Eq. (1).

In this paper, exact solutions of the one-dimensional parabolic-parabolic Keller- Segel (PP-KS) model of chemotaxis are considered. The model is made of two parabolic equations as follows

$$
\left\{\begin{array}{c}
u_{t}-u_{x x}+\left(u v_{x}\right)_{x}=0, \\
v_{t}-\alpha v_{x x}-\beta u=0 .
\end{array}\right.
$$


It is a special case of Eq. (1) when $d=\chi=1, \phi(v)=v, f(u, v)=\beta u$, here $\beta(>0)$ is a constant. By taking $\beta=1$ and introducing travelling wave transformation $y=x-c t$, (2) can be rewritten as

$$
\left\{\begin{array}{c}
u_{y}+c u-u v_{y}+\lambda=0 \\
\alpha v_{y y}+c v_{y}+u=0
\end{array}\right.
$$

where $u=u(y), v=v(y), \lambda$ is an integral constant. In [21], the authors have shown that (3) is Painleve integrable when $\alpha=2$ and soliton solutions for the particular integrable case are investigated. In this paper, more explicit exact solutions of (2) will be given. The rest of the paper is organized as follows. In section 2, exact solutions of (2) are derived by the generalized tanh function method. In section 3 , exact solutions of (2) are studied by the ( $\left.G^{\prime} / \mathrm{G}\right)$-expansion method. In section 4 , two variable-separating methods are used to get rational solutions of (2). Conclusions will be finally presented.

\section{Generalized Tanh Function Method}

Form the second equation of (3), one can easily get

$$
u=-\alpha v_{y y}-c v_{y} .
$$

Substituting (4) into the first equation of (3), one can obtain

$$
-\alpha v_{y y y}-c v_{y y}-c \alpha v_{y y}-c^{2} v_{y}+\alpha v_{y} v_{y y}+c v_{y}^{2}+\lambda=0
$$

Let $f=v_{y},(5)$ can be simplified to

$$
-\alpha f_{y y}-c f_{y}-c \alpha f_{y}-c^{2} f+\alpha f f_{y}+c f^{2}+\lambda=0
$$

From above analysis, $u$ and $v$ can be obtained by solving (6)

$$
\left\{\begin{array}{c}
v=\int f d y, \\
u=-\alpha v_{y y}-c v_{y} .
\end{array}\right.
$$

According to the main steps of the generalized tanh function method in [22], (6) is assumed to has solutions of the form

$$
f=n_{0}+\sum_{i=1}^{M} n_{i} \phi^{i}
$$

where $n_{i}(i=0,1, \ldots, M)$ are constants to be determined, $\phi=\phi(y)$ is a solution of the Riccati equation

$$
\phi_{y}=A+B \phi+N \phi^{2}
$$

Here, $A, B$ and $N$ are constants, solutions of (9) have been found in [22-23]. Balancing $f_{y y}$ with $f f_{y}$ in (6) gives $M=1$. Substituting $f=n_{0}+n_{1} \phi$ into (6) along with (9), one can get

$$
\begin{aligned}
& \alpha n_{1} N\left(n_{1}-2 N\right) \phi^{3}+\left(-3 \alpha n_{1} B N-c n_{1} N-\right. \\
& \left.c \alpha n_{1} N+\alpha n_{1} n_{0} N+\alpha n_{1}^{2} B+c n_{1}^{2}\right) \phi^{2} \\
& +\left(\alpha n_{1}^{2} A+2 c n_{1} n_{0}-\alpha n_{1} B^{2}-2 \alpha n_{1} N A\right. \\
& \left.-c n_{1} B-c \alpha n_{1} B-c^{2} n_{1}+\alpha n_{1} n_{0} B\right) \phi \\
& +\left(-\alpha B A n_{1}-c \alpha A n_{1}+\alpha n_{1} n_{0} A+\right. \\
& \left.\lambda-c n_{1} A-c^{2} n_{0}+c n_{0}^{2}\right)=0 .
\end{aligned}
$$

Setting the coefficients of $\phi^{i}(i=0,1,2,3)$ to zero, one can get

$$
\begin{aligned}
& n_{1}=2 N, n_{0}=B+c-\frac{c}{\alpha}, \\
& \alpha=2, \lambda=4 c N A+\frac{1}{4} c^{3}-c B^{2} .
\end{aligned}
$$

Taking advantage of the existing solutions of (9), one can find many kinds of travelling wave solutions for (3).

When $A=\frac{1}{2}, B=0, N=-\frac{1}{2}, \quad$ then $\lambda=-c+\frac{1}{4} c^{3}$, solutions of (6) and (3) are

$$
\begin{aligned}
& f_{1}=\frac{1}{2} c-\operatorname{coth}(y) \pm \operatorname{csch}(y) \\
& u_{1}=-\frac{1}{2} \frac{-2 c \sinh (y) \pm c^{2} \mp 4+c^{2} \cosh (y)}{\cosh (y) \pm 1} \\
& v_{1}=\frac{1}{2} c y-\ln (\sinh (y)) \pm \ln (\tanh (y))
\end{aligned}
$$

and

$$
\begin{aligned}
& f_{2}=\frac{1}{2} c-\frac{\tanh (y)}{1 \pm \operatorname{sech}(y)}, \\
& u_{2}=\frac{1}{2} \frac{-c^{2} \cosh (y)+2 c \sinh (y) \pm 4 \mp c^{2}}{\cosh (y) \pm 1}, \\
& v_{2}=\frac{1}{2} c y+\ln (\operatorname{sech}(y))-\ln (\operatorname{sech}(y) \pm 1) .
\end{aligned}
$$

When $A=\frac{1}{2}, B=0, N=\frac{1}{2}$, then $\lambda=c+\frac{1}{4} c^{3}$, solutions of (6) and (3) are

$$
\begin{aligned}
& f_{3}=\frac{1}{2} c+\tan (y) \pm \sec (y), \\
& u_{3}=\frac{1}{2} \frac{2 c \cos (y)-c^{2} \sin (y) \pm c^{2} \pm 4}{\sin (y) \mp 1}, \\
& v_{3}=\frac{1}{2} c y-\ln (\cos (y)) \pm \ln (\tan (y)+\sec (y)),
\end{aligned}
$$

and 


$$
\begin{aligned}
& f_{4}=\frac{1}{2} c+\csc (y)-\cot (y), \\
& u_{4}=-\frac{1}{2} \frac{c^{2} \cos (y)+2 c \sin (y)+c^{2}+4}{1+\cos (y)}, \\
& v_{4}=\frac{1}{2} c y-\ln (\csc (y)+\cot (y))-\ln (\sin (y)) .
\end{aligned}
$$

When $\quad A=-\frac{1}{2}, B=0, N=-\frac{1}{2}, \quad$ then $\quad \lambda=c+\frac{1}{4} c^{3}$, solutions of (6) and (3) are

$$
\begin{aligned}
& f_{5}=\frac{1}{2} c-\csc (y)-\cot (y), \\
& u_{5}=-\frac{1}{2} \frac{c^{2} \cos (y)+2 c \sin (y)-c^{2}-4}{-1+\cos (y)}, \\
& v_{5}=\frac{1}{2} c y+\ln (\csc (y)+\cot (y))-\ln (\sin (y)) .
\end{aligned}
$$

When $\quad A=1, B=0, N=-1, \quad$ then $\quad \lambda=-4 c+\frac{1}{4} c^{3}$, solutions of (6) and (3) are

$$
\begin{aligned}
& f_{6}=\frac{1}{2} c-2 \tanh (y), \\
& u_{6}=\frac{4 c \cosh (y) \sinh (y)-c^{2} \cosh ^{2}(y)+8}{2 \cosh ^{2}(y)}, \\
& v_{6}=\frac{1}{2} c y-2 \ln (\cosh (y)),
\end{aligned}
$$

and

$$
\begin{aligned}
& f_{7}=\frac{1}{2} c-2 \operatorname{coth}(y), \\
& u_{7}=\frac{4 c \cosh (y) \sinh (y)-c^{2} \cosh (y)-8+c^{2}}{2 \sinh ^{2}(y)}, \\
& v_{7}=\frac{1}{2} c y-2 \ln (\sinh (y)) .
\end{aligned}
$$

When $A=1, B=0, N=1$, then $\lambda=4 c+\frac{1}{4} c^{3}$, solutions of (6) and (3) are

$$
\begin{aligned}
& f_{8}=\frac{1}{2} c+2 \tan (y) \\
& u_{8}=\frac{4 c \cos (y) \sin (y)+c^{2} \cos ^{2}(y)+8}{-2 \cos ^{2}(y)} \\
& v_{8}=\frac{1}{2} c y-2 \ln (\cos (y))
\end{aligned}
$$

When $A=-1, B=0, N=-1, \quad$ then $\quad \lambda=4 c+\frac{1}{4} c^{3}$, solutions of (6) and (3) are

$$
\begin{aligned}
& f_{9}=\frac{1}{2} c-2 \cot (y), \\
& u_{9}=\frac{4 c \cos (y) \sin (y)+c^{2} \cos ^{2}(y)-8-c^{2}}{-2\left(-1+\cos ^{2}(y)\right)}, \\
& v_{9}=\frac{1}{2} c y-2 \ln (\sin (y)) .
\end{aligned}
$$

When $A=1, B=-2, N=2, \quad$ then $\quad \lambda=4 c+\frac{1}{4} c^{3}$, solutions of (6) and (3) are

$$
\begin{aligned}
& f_{10}=-2+\frac{1}{2} c+\frac{4 \tan (y)}{1+\tan (y)}, \\
& u_{10}=\frac{2 c^{2} \cos (y) \sin (y)-8 c \cos ^{2}(y)+16+c^{2}+4 c}{-2(1+2 \cos (y) \sin (y))}, \\
& v_{10}=\frac{1}{2} c y+\ln \left(1+\tan ^{2}(y)\right)-2 \ln (1+\tan (y)) .
\end{aligned}
$$

When $A=1, B=2, N=2$, then $\lambda=4 c+\frac{1}{4} c^{3}$, solutions of (6) and (3) are

$$
\begin{aligned}
& f_{11}=2+\frac{1}{2} c+\frac{4 \tan (y)}{1-\tan (y)}, \\
& u_{11}=\frac{2 c^{2} \cos (y) \sin (y)-8 c \cos ^{2}(y)-16-c^{2}+4 c}{-2(-1+2 \cos (y) \sin (y))}, \\
& v_{11}=\frac{1}{2} c y+\ln \left(1+\tan ^{2}(y)\right)-2 \ln (-1+\tan (y)) .
\end{aligned}
$$

When $A=-1, B=2, N=-2$, then $\lambda=4 c+\frac{1}{4} c^{3}$, solutions of (6) and (3) are

$$
f_{12}=2+\frac{1}{2} c-\frac{4 \cot (y)}{1+\cot (y)},
$$

$$
\begin{aligned}
& u_{12}=\frac{2 c^{2} \cos (y) \sin (y)-8 c \cos ^{2}(y)-16-c^{2}+4 c}{-2(-1+2 \cos (y) \sin (y))}, \\
& v_{12}=\frac{1}{2} c y+\ln \left(1+\cot ^{2}(y)\right)-2 \ln (1+\cot (y))-\pi .
\end{aligned}
$$

When $A=-1, B=-2, N=-2, \quad$ then $\quad \lambda=4 c+\frac{1}{4} c^{3}$, solutions of (6) and (3) are 


$$
\begin{aligned}
& f_{13}=-2+\frac{1}{2} c-\frac{4 \cot (y)}{1-\cot (y)}, \\
& u_{13}=\frac{-2 c^{2} \cos (y) \sin (y)+8 c \cos ^{2}(y)+16+c^{2}-4 c}{2(-1+2 \cos (y) \sin (y))}, \\
& v_{13}=\frac{1}{2} c y+\ln \left(1+\cot ^{2}(y)\right)-2 \ln (-1+\cot (y))-\pi .
\end{aligned}
$$

When $A=0, B=0, N \neq 0$, then $\lambda=\frac{1}{4} c^{3}$, solutions of (6) and (3) are

$$
\begin{aligned}
& f_{14}=\frac{1}{2} c-\frac{2 N}{N y+N_{1}}, \\
& u_{14}=\frac{8 N^{2}+c^{2} N^{2} y^{2}+2 c^{2} N N_{1} y+c^{2} N_{1}^{2}-4 c N^{2} y-4 c N_{1} N}{-2\left(N y+N_{1}\right)^{2}}, \\
& v_{14}=\frac{1}{2} c y-2 \ln \left(N y+N_{1}\right),
\end{aligned}
$$

where $N_{1}$ is a constant.

When $A=1, B=0, N=-4, \quad$ then $\quad \lambda=-16 c+\frac{1}{4} c^{3}$, solutions of (6) and (3) are

$$
\begin{aligned}
f_{15}= & \frac{1}{2} c-\frac{8 \tanh (y)}{1+\tanh ^{2}(y)}, \\
u_{15}= & \frac{1}{-2\left(1+\tanh ^{2}(y)\right)^{2}}\left(-32+64 \tanh ^{2}(y)-\right. \\
& 32 \tanh ^{4}(y)+c^{2}+2 c^{2} \tanh ^{2}(y)+c^{2} \tanh ^{4}(y) \\
& \left.-16 c \tanh (y)-16 c^{3} \tanh (y)\right), \\
v_{15}= & \frac{1}{2} c y-2 \ln \left(1+\tanh ^{2}(y)\right)+ \\
& 2 \ln (\tanh (y)-1)+2 \ln (\tanh (y)+1) .
\end{aligned}
$$

When $A=1, B=0, N=4, \quad$ then $\quad \lambda=16 c+\frac{1}{4} c^{3}$, solutions of (6) and (3) are

$$
\begin{aligned}
& f_{16}=\frac{1}{2} c+\frac{8 \tan (y)}{1-\tan ^{2}(y)}, \\
& u_{16}=\frac{-1}{2\left(2 \cos ^{2}(y)-1\right)^{2}}\left(1 6 c \operatorname { s i n } ( y ) \operatorname { c o s } ( y ) \left(2 \cos ^{2}(y)\right.\right. \\
& \left.-1)-4 c^{2} \cos ^{2}(y) \sin ^{2}(y)+32+c^{2}\right), \\
& v_{16}=\frac{1}{2} c y+2 \ln \left(1+\tan ^{2}(y)\right)-2 \ln (\tan (y)+1) \\
& -2 \ln (\tan (y)-1) .
\end{aligned}
$$

When $A=-1, B=0, N=-4, \quad$ then $\quad \lambda=16 c+\frac{1}{4} c^{3}$, solutions of (6) and (3) are

$$
\begin{aligned}
f_{17}= & \frac{1}{2} c-\frac{8 \cot (y)}{1-\cot ^{2}(y)} \\
u_{17}= & \frac{-1}{2\left(2 \cos ^{2}(y)-1\right)^{2}}\left(16 c \sin (y) \cos (y)\left(2 \cos ^{2}(y)-1\right)\right. \\
& \left.-4 c^{2} \cos ^{2}(y) \sin ^{2}(y)+32+c^{2}\right) \\
v_{17}= & \frac{1}{2} c y+2 \ln \left(1+\cot ^{2}(y)\right)-2 \ln (\cot (y)+1) \\
& -2 \ln (\cot (y)-1)
\end{aligned}
$$

\section{3. $\left(G^{\prime} / G\right)$-Expansion Method}

In this section, (6) is solved by the $\left(G^{\prime} / G\right)$-expansion method and solutions of (3) can be obtained by (7). According to the $\left(G^{\prime} / G\right)$-expansion method [24-25], the function $f$ is expressed as a polynomial in $\left(G^{\prime} / G\right)$

$$
f=m_{0}+\sum_{i=1}^{M} m_{i}\left(\frac{G^{\prime}}{G}\right)^{i},
$$

where $m_{i}(i=0,1, \ldots, M)$ are constants to be determined, $G=G(y) \quad$ satisfies a second-order linear ordinary differential equation

$$
G^{\prime \prime}+r G^{\prime}+\mu G=0
$$

Here, $r$ and $\mu$ are constants, solutions of (13) have been found in [24-25]. Balancing $f_{y y}$ with $f f_{y}$ in (6) gives $M=1$. Substituting $f=m_{0}+m_{1}\left(\frac{G^{\prime}}{G}\right)$ into (6) along with (13), one can get

$$
\begin{aligned}
& -\alpha m_{1}\left(m_{1}+2\right)\left(\frac{G^{\prime}}{G}\right)^{3}-\alpha m_{1}\left(3 r-\frac{c}{\alpha}-c+m_{0}+m_{1} r-\frac{m_{1} c}{\alpha}\right) \\
& \left(\frac{G^{\prime}}{G}\right)^{2}+\left(-\alpha m_{1} r^{2}-2 \alpha m_{1} \mu+c m_{1} r+c \alpha m_{1} r-c^{2} m_{1}-\right. \\
& \left.\alpha m_{1} m_{0} r-\alpha m_{1}^{2} \mu\right)\left(\frac{G^{\prime}}{G}\right)+\left(\lambda-c^{2} m_{0}+c m_{0}^{2}+c m_{1} \mu\right. \\
& \left.-\alpha m_{1} r \mu+c \alpha m_{1} \mu-\alpha m_{1} m_{0} \mu\right)=0 .
\end{aligned}
$$

Setting the coefficients of $\left(\frac{G^{\prime}}{G}\right)^{i}(i=0,1,2,3)$ to zero, one can get

$$
\begin{aligned}
& m_{1}=-2, \alpha=2, m_{0}=r-\frac{c}{2}, \\
& \lambda=4 c \mu+\frac{1}{4} c^{3}-c r^{2} .
\end{aligned}
$$

Taking advantage of exact solutions of (13), two kinds of exact travelling wave solutions for (3) can be found.

When $r^{2}-4 \mu>0$, solutions for (6) and (3) are as follows 


$$
\begin{aligned}
& f_{18}=\frac{1}{2} c-\sqrt{r^{2}-4 \mu} \frac{\left(C_{1} \sinh \left(\frac{1}{2} \sqrt{r^{2}-4 \mu} y\right)+C_{2} \cosh \left(\frac{1}{2} \sqrt{r^{2}-4 \mu} y\right)\right)}{\left(C_{1} \cosh \left(\frac{1}{2} \sqrt{r^{2}-4 \mu} y\right)+C_{2} \sinh \left(\frac{1}{2} \sqrt{r^{2}-4 \mu} y\right)\right.}, \\
& u_{18}=\frac{1}{2\left(C_{1} \cosh \left(\frac{1}{2} \sqrt{r^{2}-4 \mu} y\right)+C_{2} \sinh \left(\frac{1}{2} \sqrt{r^{2}-4 \mu} y\right)\right)^{2}}\left(-2 r^{2} C_{2}^{2}+2 r^{2} C_{1}^{2}+8 \mu C_{2}^{2}-8 \mu C_{1}^{2}+c^{2} C_{2}^{2}\right. \\
& -2 c C_{1} C_{2} \sqrt{r^{2}-4 \mu}-c^{2} C_{1}^{2} \cosh ^{2}\left(\frac{1}{2} \sqrt{r^{2}-4 \mu} y\right)-2 c^{2} C_{1} C_{2} \cosh \left(\frac{1}{2} \sqrt{r^{2}-4 \mu} y\right) \sinh \left(\frac{1}{2} \sqrt{r^{2}-4 \mu y}\right) \\
& -c^{2} C_{2}^{2} \cosh ^{2}\left(\frac{1}{2} \sqrt{r^{2}-4 \mu} y\right)+2 c C_{1}^{2} \sqrt{r^{2}-4 \mu} \cosh \left(\frac{1}{2} \sqrt{r^{2}-4 \mu} y\right) \sinh \left(\frac{1}{2} \sqrt{r^{2}-4 \mu} y\right) \\
& +4 c C_{1} C_{2} \sqrt{r^{2}-4 \mu} \cosh \left(\frac{1}{2} \sqrt{r^{2}-4 \mu} y\right)+2 c C_{2}^{2} \sqrt{r^{2}-4 \mu} \cosh \left(\frac{1}{2} \sqrt{r^{2}-4 \mu} y\right) \sinh \left(\frac{1}{2} \sqrt{\left.\left.r^{2}-4 \mu y\right)\right)}\right. \\
& v_{18}=\frac{1}{2} c y-2 \ln \left(C_{1} \cosh \left(\frac{1}{2} \sqrt{r^{2}-4 \mu} y\right)+C_{2} \sinh \left(\frac{1}{2} \sqrt{r^{2}-4 \mu} y\right)\right)
\end{aligned}
$$

When $r^{2}-4 \mu<0$, solutions for (6) and (3) are as follows

$$
\begin{aligned}
& f_{19}=\frac{1}{2} c-\sqrt{4 \mu-r^{2}} \frac{\left(-C_{3} \sin \left(\frac{1}{2} \sqrt{4 \mu-r^{2}} y\right)+C_{4} \cos \left(\frac{1}{2} \sqrt{4 \mu-r^{2}} y\right)\right)}{\left(C_{3} \cos \left(\frac{1}{2} \sqrt{4 \mu-r^{2}} y\right)+C_{4} \sin \left(\frac{1}{2} \sqrt{4 \mu-r^{2}} y\right)\right)}, \\
& u_{19}=\frac{1}{2\left(\left(C_{3}^{2}+C_{4}^{2}\right) \cos ^{2}\left(\frac{1}{2} \sqrt{4 \mu-r^{2}} y\right)+2 C_{3} C_{4} \cos \left(\frac{1}{2} \sqrt{4 \mu-r^{2}} y\right) \sin \left(\frac{1}{2} \sqrt{4 \mu-r^{2}} y\right)+C_{4}^{2}\right)^{2}} \\
& \left(2 r^{2} C_{4}^{2}-8 \mu C_{4}^{2}+2 r^{2} C_{3}^{2}-8 \mu C_{3}^{2}-c^{2} C_{4}^{2}-2 c C_{3} C_{4} \sqrt{4 \mu-r^{2}}-c^{2} C_{3}^{2} \cos ^{2}\left(\frac{1}{2} \sqrt{4 \mu-r^{2}} y\right)\right. \\
& -2 c^{2} C_{3} C_{4} \cos \left(\frac{1}{2} \sqrt{4 \mu-r^{2}} y\right) \sin \left(\frac{1}{2} \sqrt{4 \mu-r^{2}} y\right)+c^{2} C_{4}^{2} \cos ^{2}\left(\frac{1}{2} \sqrt{4 \mu-r^{2}} y\right) \\
& -2 c C_{3}^{2} \sqrt{4 \mu-r^{2}} \cos \left(\frac{1}{2} \sqrt{4 \mu-r^{2}} y\right) \sin \left(\frac{1}{2} \sqrt{4 \mu-r^{2}} y\right)+4 c C_{3} C_{4} \sqrt{4 \mu-r^{2}} \cos ^{2}\left(\frac{1}{2} \sqrt{4 \mu-r^{2}} y\right) \\
& \left.+2 c C_{4}^{2} \sqrt{4 \mu-r^{2}} \cos \left(\frac{1}{2} \sqrt{4 \mu-r^{2}} y\right) \sin \left(\frac{1}{2} \sqrt{4 \mu-r^{2}} y\right)\right), \\
& v_{19}=\frac{1}{2} c y-2 \ln \left(C_{3} \cos \left(\frac{1}{2} \sqrt{4 \mu-r^{2}} y\right)+C_{4} \sin \left(\frac{1}{2} \sqrt{4 \mu-r^{2}} y\right)\right)
\end{aligned}
$$

where $C_{i}(i=1,2,3,4)$ are constant.

Remark 1 When $r^{2}-4 \mu=4, C_{1}=1, C_{2}=0$,

$$
\begin{aligned}
& f_{18}=\frac{1}{2} c-2 \tanh (y), \\
& u_{18}=\frac{1}{2 \cosh ^{2}(y)}\left(8-c^{2} \cosh ^{2}(y)+4 c \cosh (y) \sinh (y)\right), \\
& v_{18}=\frac{1}{2} c y-2 \ln (\cosh (y)),
\end{aligned}
$$

this is exactly the same with the solution $\left(f_{6}, u_{6}, v_{6}\right)$ obtained in section 2. Similarly, when $r^{2}-4 \mu=4, C_{1}=0, C_{2}=1$, the solution $\left(f_{18}, u_{18}, v_{18}\right)$ is the same with $\left(f_{7}, u_{7}, v_{7}\right)$, when $r^{2}-4 \mu=-4, C_{3}=1, C_{4}=0$, the solution $\left(f_{19}, u_{19}, v_{19}\right)$ is the same with $\left(f_{8}, u_{8}, v_{8}\right)$, when $\quad r^{2}-4 \mu=-4, C_{3}=0, C_{4}=1$, the solution $\left(f_{19}, u_{19}, v_{19}\right)$ is the same with $\left(f_{9}, u_{9}, v_{9}\right)$. When $r^{2}-4 \mu, C_{1}$ and $C_{2}$ take other values, the solutions obtained in this section are completely new.

Remark 2 The solutions $\left(f_{i}, u_{i}, v_{i}\right)(i=1,2, \ldots, 19)$ are all travelling wave solutions for the particular integrable case of (3) when $\alpha=2$. The correctness of them have been checked by Maple. 


\section{Variable-Separating Method}

Variable-separating method is a classical method to solve partial differential equations. In [26], the author proposed a new variable-separating method. In the following (2) will be solved by the two variable-separating methods.

Form the second equation of (2), one can easily get

$$
u=-\frac{1}{\beta}\left(v_{t}-\alpha v_{x x}\right) .
$$

Substituting (18) into the first equation of (2), one can obtain

$$
\begin{aligned}
& v_{t t}-\alpha v_{x x t}-v_{x x t}+\alpha v_{x x x x}+v_{x} v_{x t} \\
& -\alpha v_{x} v_{x x x}+v_{t} v_{x x}-\alpha v_{x x}^{2}=0 .
\end{aligned}
$$
(2).

Obviously, one only need to solve (19) instead of solving

\subsection{New Variable-Separating Method}

According to the new variable-separating method, one may assume

$$
v=Q+M
$$

where $Q=Q(x), M=M(t)$. Substituting (20) into (19), one can get

$$
M_{t t}+\alpha Q_{x x x x}-\alpha Q_{x} Q_{x x x}+Q_{x x} M_{t}-\alpha Q_{x x}{ }^{2}=0 .
$$

Let

$$
Q=G_{1} x^{2}+G_{2} x+G_{3},
$$

where $G_{i}(i=1,2,3)$ are constants. Substituting (22) into (21), one can get

$$
M_{t t}+2 G_{1} M_{t}-4 \alpha G_{1}^{2}=0
$$

(23) is an ordinary differential equation, it has solutions

$$
M=\frac{G_{4}}{-2 G_{1}} e^{\left(-2 G_{1} t\right)}+2 \alpha G_{1} t+G_{5},
$$

where $G_{4}$ and $G_{5}$ are constants. Therefore, the solution of (2) are obtained as

$$
\begin{aligned}
& u=\frac{G_{4}}{\beta} e^{\left(-2 G_{1} t\right)}, \\
& v=G_{1} x^{2}+G_{2} x+G_{3}-\frac{G_{4}}{2 G_{1}} e^{\left(-2 G_{1} t\right)}+2 \alpha G_{1} t+G_{5} .
\end{aligned}
$$

The solution (24) is different with the solutions $\left(f_{i}, u_{i}, v_{i}\right)(i=1,2, \ldots, 19)$ in section 2 and 3 , since it is not travelling wave solutions. In addition, (24) is satisfied for all values of $\alpha$ while the travelling wave solutions $\left(f_{i}, u_{i}, v_{i}\right)(i=1,2, \ldots, 19)$ are only satisfied for $\alpha=2$. That is to say, $\left(f_{i}, u_{i}, v_{i}\right)(i=1,2, \ldots, 19)$ are solutions of (2) when it is Painleve integrable, and (24) is a solution of (2) whether it is Painleve integrable or not.

\subsection{Classical Variable-Separating Method}

According to the classical variable-separating method, let

$$
v=Q M
$$

where $Q=Q(x), M=M(t)$. Substituting (25) into (19), one gets

$$
\begin{aligned}
& Q M_{t t}-Q_{x x} M_{t}(\alpha+1)+\alpha Q_{x x x x} M- \\
& \alpha M^{2}\left(Q_{x} Q_{x x x}+Q_{x x}{ }^{2}\right)+M M_{t}\left(Q_{x}{ }^{2}+Q Q_{x x}\right)=0 .
\end{aligned}
$$

It is difficult to give all explicit solutions of (26), so some particular cases are given.

Let

$$
Q=e^{G_{6} x}
$$

where $G_{6}$ is constant. Substituting (27) into (26), one can get

$$
\begin{aligned}
& 2 G_{6}^{2} M\left(M_{t}-\alpha G_{6}^{2} M\right) e^{2 G_{6} x}+ \\
& e^{G_{6} x}\left(-G_{6}^{2}\left(M_{t}-\alpha G_{6}^{2} M\right)-\left(M_{t}-\alpha G_{6}^{2} M\right)_{t}\right)=0 .
\end{aligned}
$$

From (28),

$$
M=G_{7} e^{\left(\alpha G_{6}^{2} t\right)}
$$

where $G_{7}$ is constant. Therefore, a solution of (2) is obtained as

$$
\begin{aligned}
& u=0, \\
& v=G_{7} e^{\left(\alpha G_{6}{ }^{2} t+G_{6} x\right)} .
\end{aligned}
$$

Let $Q=G_{8}$ in (26), then

$$
M=G_{9} t+G_{10},
$$

where $G_{i}(i=8,9,10)$ are constants. Then a solution of $(2)$ is obtained as

$$
\begin{aligned}
& u=\frac{G_{8} G_{9}}{\beta}, \\
& v=G_{8}\left(G_{9} t+G_{10}\right) .
\end{aligned}
$$

Let $M=1$ in (26), one can get

$$
\alpha Q_{x x x x}-\alpha\left(Q_{x} Q_{x x x}+Q_{x x}^{2}\right)=0
$$

From (32), 


$$
Q=-2 \ln \left(-\frac{1}{2} G_{11} x-\frac{1}{2} G_{12}\right)
$$

Then a solution of (2) is obtained as

$$
\begin{aligned}
& u=\frac{-2 \alpha G_{11}^{2}}{\left(G_{11} x+G_{12}\right)^{2} \beta}, \\
& v=-2 \ln \left(-\frac{1}{2} G_{11} x-\frac{1}{2} G_{12}\right),
\end{aligned}
$$

where $G_{11}$ and $G_{12}$ are constants, $-\frac{1}{2} G_{11} x-\frac{1}{2} G_{12}>0$.

Remark 3 The solutions (29), (31) and (33) are satisfied for all values of $\alpha$ in (2). Although the expressions of (29), (31) and (33) are simple, they all reflect different phenomenon of chemotaxis. For example, (31) describes a particular case when the cell density $u$ is unchanging, the corresponding concentration of the chemical substance $v$ is a linear function of time $t$.

Remark 4 Compared with the work in [21], more new exact solutions for the particular integrable case when $\alpha=2$ are given. Furthermore, exact solutions for the general case $\alpha>0$ are obtained. In addition, all the obtained solutions have explicit expressions, so they are easier to use.

\section{Conclusion}

A mathematical model of chemotaxis (the movement of biological cells or organisms in response to chemical gradients) named as parabolic - parabolic Keller-Segel (PP-KS) equation is considered in this paper. By using the generalized tanh function method and $\left(G^{\prime} / \mathrm{G}\right)$-expansion method, plenty of new travelling solutions are obtained for the particular integrable case $(\alpha=2)$ of the PP-KS model (2). These solutions contain hyperbolic function solutions, triangular periodic solutions and rational function solutions. By using classical variable-separating method and new variable-separating method, a lot of algebraically explicit analytical solutions are obtained for the general case $(\alpha>0)$ of the PP-KS model (2). Compared with the results in [21], more new exact solutions for the PP-KS model have been derived whether it is Painleve integrable or not, and the obtained solutions in this paper all have explicit expressions. They can be used in numerical simulation and help one to understand the mechanism reflected by PP-KS model. In the future, exact solutions of the generalizations of the KS model will be studied since they play critical roles in a wide range of biological phenomena.

\section{Acknowledgements}

The work is supported by the Natural Science Foundation of Shandong Province (ZR2017LA012), Science and Technology Program of Colleges and Universities in Shandong (J17KA156), and National Natural Science Foundation of China (11501082).

\section{References}

[1] R. Hirota and J. Satsuma, "Soliton solutions of a coupled Korteweg-de Vries equation," Phys. Lett. A, 1981, 85, 407408 .

[2] M. J. Ablowitz and H. Segur, "Soliton and the inverse scattering transformation," SIAM, Philadelphia, PA, 1981.

[3] M. Wadati, "Wave propagation in nonlinear lattice. I," J. Phys. Soc. Jpn., 1975, 38, 673-680.

[4] R. Conte and M. Musette, "Painleve analysis and Backlund transformation in the Kuramoto-Sivashinsky equation," J. Phys. A: Math. Gen., 1989, 22, 169-177.

[5] L. H. Zhang, X. Q. Liu and C. L. Bai, "New multiple soliton-like and periodic solutions for (2+1)-dimensional canonical generalized KP equation with variable coefficients," Commun. Theor. Phys., 2006, 46, 793-798.

[6] C. L. Bai, C. J. Bai and H. Zhao, "A new generalized algebraic method and its application in nonlinear evolution equations with variable coefficients," Z. Naturforsch. A, 2005, 60, 211220 .

[7] G. W. Wang, "Symmetry analysis and rogue wave solutions for the (2+1)-dimensional nonlinear Schrödinger equation with variable coefficients," Appl. Math. Lett., 2016, 56, 5664.

[8] H. Z. Liu and Y. X. Geng, "Symmetry reductions and exact solutions to the systems of carbon nanotubes conveying fluid," J. Differential Equations, 2013, 254, 2289-2303.

[9] E. G. Fan, "Uniformly constructing a series of explicit exact solutions to nonlinear equations in mathematical physics," Chaos, Solitons and Fractals, 2003, 16, 819-839.

[10] D. S. Wang and H. B. Li, "Elliptic equation's new solutions and their applications to two nonlinear partial differential equations," Appl. Math. Comput., 2007, 188, 762-771.

[11] E. F. Keller and L. A. Segel, "Initiation of slime mold aggregation viewed as an instability," J. Theor. Biol., 1970, 26, 399-415.

[12] E. F. Keller and L. A. Segel, "Model for chemotaxis," J. Theor. Biol., 1971, 30, 225-234.

[13] E. F. Keller and L. A. Segel, "Traveling bands of chemotactic bacteria: A theorectical analysis," J. Theor. Biol., 1971, 26, 235-248.

[14] J. Adler, "Chemotaxis in bacteria," Science, 1966, 153, 708716.

[15] J. Adler, "Chemoreceptors in bacteria," Science, 1969, 166, 1588-1597.

[16] Z. A. Wang, "Mathematics of traveling waves in chemotaxis," Discrete Cont. Dyn. B, 2013, 18, 601-641.

[17] D. Horstmann, "From 1970 until present: the Keller-Segel model in chemotaxis and its consequences. II," Jahresber. Deutsch. Math.-Verein., 2004, 106, 51-69.

[18] T. Hillen and K. J. Painter, "A user's guide to PDE models for chemotaxis,” J. Math. Biol., 2009, 58, 183-217. 
[19] D. Horstmann, "From 1970 until present: the Keller-Segel model in chemotaxis and its consequences, I," Jahresber. Deutsch. Math.-Verein., 2003, 105, 103-165.

[20] J. Murray, "Mathematical Biology: II. Spatial Models and Biomedical Applications," 3rd edition, Springer, New York, 2003.

[21] M. Shubina, "The 1D parabolic-parabolic Patlak-Keller-Segel model of chemotaxis: the particular integrable case and soliton solution," J. Math. Phys., 2016, 57, 091501.

[22] H. T. Chen and H. Q. Zhang, "New double periodic and multiple soliton solutions of the generalized (2+1)-dimensional Boussinesq equation," Chaos Solitons and Fractals, 2004, 20, 765-769.

[23] L. H. Zhang, L. H. Dong and L. M. Yan, "Construction of non-travelling wave solutions for the generalized variable-coefficient Gardner equation," Appl. Math. Comput., 2008, 203, 784-791.

[24] M. L. Wang, X. Z. Li and J. L. Zhang, "The (G '/G)-expansion method and travelling wave solutions of nonlinear evolution equations in mathematical physics," Phys. Lett. A, 2008, 372, 417-423.

[25] G. W. Wang, T. Z. Xu, R. Abazari, Z. Jovanoski and A. Biswas, "Shock waves and other solutions to the Benjamin-Bona-Mahoney-Burgers equation with dual power law nonlinearity," Acta. Phys. Pol. A, 2014, 126, 1221-1225.

[26] R. X. Cai, Q. B. Liu, “A new method for deriving analytical solutions of partial differential equations algebraically explicit analytical solutions of two-buoyancy natural convection in porous media," Sci. China. Ser. G, 2008, 51, 1733-1744. 